\title{
Proteomics of stress responses in wheat and barley-search for potential protein markers of stress tolerance
}

\section{Klára Kosová *, Pavel Vítámvás and Ilja T. Prášil}

Laboratory of Plant Stress Biology and Biotechnology, Division of Crop Genetics and Breeding, Department of Plant Genetics, Breeding and Product Quality, Crop Research Institute, Prague, Czech Republic

\section{Edited by:}

Jesus V. Jorrin Novo, University of

Cordoba, Spain

Reviewed by:

Martin Hajduch, Slovak Academy of Sciences, Slovakia

Jesus V. Jorrin Novo, University of Cordoba, Spain

Arkadiusz Kosmala, Institute of Plant Genetics of the Polish Academy of Sciences, Poland

*Correspondence:

Klára Kosová, Laboratory of Plant Stress Biology and Biotechnology, Division of Crop Genetics and Breeding, Crop Research Institute, Drnovská 507, Prague 6 - Ruzyně, 161 06, Czech Republic e-mail:kosova@vurv.cz
Wheat (Triticum aestivum; T. durum) and barley (Hordeum vulgare) agricultural production is severely limited by various abiotic and biotic stress factors. Proteins are directly involved in plant stress response so it is important to study proteome changes under various stress conditions. Generally, both abiotic and biotic stress factors induce profound alterations in protein network covering signaling, energy metabolism (glycolysis, Krebs cycle, ATP biosynthesis, photosynthesis), storage proteins, protein metabolism, several other biosynthetic pathways (e.g., S-adenosylmethionine metabolism, lignin metabolism), transport proteins, proteins involved in protein folding and chaperone activities, other protective proteins (LEA, PR proteins), ROS scavenging enzymes as well as proteins affecting regulation of plant growth and development. Proteins which have been reported to reveal significant differences in their relative abundance or posttranslational modifications between wheat, barley or related species genotypes under stress conditions are listed and their potential role in underlying the differential stress response is discussed. In conclusion, potential future roles of the results of proteomic studies in practical applications such as breeding for an enhanced stress tolerance and the possibilities to test and use protein markers in the breeding are suggested.

Keywords: proteome, barley, wheat, abiotic stress factors, biotic stress factors, protein markers

\section{INTRODUCTION}

Wheat (Triticum aestivum; T. durum) and barley (Hordeum vulgare) represent major cereal crops grown in temperate climate areas. Cereal agricultural production is limited by a wide array of abiotic and biotic stress factors including drought (Cattivelli et al., 2008), cold (Thomashow, 1999; Kosová et al., 2008a), heat, salinity (Munns, 2005; Kosová et al., 2013a), imbalances in mineral nutrition, viral (Kosová et al., 2008b) and fungal pathogens such as Fusarium (Kosová et al., 2009; Yang et al., 2010a,b), leaf rust (Puccinia triticina; Rampitsch et al., 2006), blotch (Septoria tritici; Yang et al., 2013) and others, often acting in combinations under field conditions (Mittler, 2006). Proteome plays an important role in stress response since proteins are directly involved in several processes aimed at an enhancement of stress tolerance being "closer to phenotype" than transcripts.

During the past decades, the boom of high-throughput proteomics techniques has enabled the researchers to study plant proteome responses to various factors including stresses in a complex way. Despite numerous studies reporting identifications of a few thousand of proteins in plant samples, a complete description of plant proteome in a given tissue, developmental phase and environmental conditions still remains a great challenge (Jorrin-Novo et al., 2009).

Both abiotic and biotic stresses induce profound changes in plant proteomes aimed at an adjustment of metabolism to altered environment and an enhancement of plant stress tolerance. Plant stress response is a dynamic process and several phases with a unique proteome composition could be distinguished (Levitt, 1980; Larcher, 2003). Reviews on plant proteome responses to abiotic stresses (Kosová et al., 2011; Hossain et al., 2012) and pathogens (Sergeant and Renaut, 2010; Gonzalez-Fernandez and Jorrin-Novo, 2012) provide important overviews; however, several novel studies were published recently (Table 1).

Most proteomic papers aimed at an investigation of plant stress responses are comparative studies that are based on comparison of proteome composition in stressed plants vs. control ones, and also in differentially-tolerant genotypes exposed to stress. Moreover, studies on the roles of subcellular proteomes such as chloroplast (Kamal et al., 2012) and mitochondrial (Jacoby et al., 2010, 2013) proteomes as well as posttranslational modifications (PTMs) such as phosphoproteomics (Yang et al., 2013; Zhang et al., 2014) in wheat exposed to stress have been published recently. Considering the increasing amount of proteomic data, it is arising necessary to mine the data published in various proteomic studies in order to identify key proteins involved in plant responses to a wide array of stress factors (dehydrative stresses - drought, osmotic stress, salinity, frost, heat) as well as proteins induced only at specific stress conditions (e.g., phytochelatins and heavy metal stress). An attempt has already been published regarding proteomic studies under salinity (Zhang et al., 2012). Moreover, comparison of proteome responses in 
Table 1 | Continued

\begin{tabular}{|c|c|c|c|c|}
\hline Plant material & Treatment & Methods & $\begin{array}{l}\text { Major differentially- } \\
\text { abundant proteins }\end{array}$ & References \\
\hline
\end{tabular}

\section{HEAT}

(DAP)

\begin{tabular}{|c|c|c|}
\hline $\begin{array}{l}\text { Common wheat-Fang } \\
\text { (T), Wyuna (S)-grain } \\
\text { endosperm }\end{array}$ & $\begin{array}{l}40 / 25^{\circ} \mathrm{C} \\
\text { (day/night)-15, 16, } 17 \\
\text { days post-anthesis }\end{array}$ & $\begin{array}{l}\text { TCA/acetone 2DE } \\
\text { MALDI-TOF; MS/MS } \\
\text { Q-TOF }\end{array}$ \\
\hline $\begin{array}{l}\text { Common } \\
\text { wheat-Thésée-grain }\end{array}$ & $\begin{array}{l}34 / 10^{\circ} \mathrm{C} \\
\text { (day/night)-697 and } \\
763^{\circ} \mathrm{C} \mathrm{d} \\
\text { (degree-days) }\end{array}$ & $\begin{array}{l}\text { Sodium-phosphate } \\
\text { buffer; } 2 \text { DE MALDI-TOF }\end{array}$ \\
\hline
\end{tabular}

V-ATPase; eEF-Tu, CPN60, 60S and

$40 S$ ribosomal proteins; GS

Genotypic differences: Seven small

Skylas et al., 2002 HSP ( $16.9 \mathrm{kD}$ class I HSP) proteins unique to $T$

42 identified proteins Majoul et al., 2004

Up: 20 kD sHSP, 17 kD class II HSP;

HSP82 (HSP90 family); eEF-Tu,

V-ATPase subunit E

Down: starch biosynthesis enzymes granule-bound starch synthase, glucose-1-phosphate adenyltransferase; $\beta$-amylase; $\beta$ subunit ATP synthase

\section{DROUGHT}

Common wheat-spring wheats Arvand, Khazar-1, Kelk Afghani-grain Australian wheats Kukri (S), Excalibur, RAC875 (T)-leaf

\section{Field conditions \\ (Azarbayjan) plus artificial irrigation Water witholding until leaf wilting in Kukri (S)-14, 24 days, and rewatering (25 days)}

\section{DE MALDI-TOF/TOF}

TCA; nanoLC-MS/MS iTRAO 8plex

\section{1 (57 identified) \\ Up: Trx h, 1-Cys peroxiredoxin, GST; 2007 \\ PDI; LEA, sHSP17, HSP70}

1299 identified proteins $\quad$ Ford et al., 2011

Increase in ROS

metabolism-associated proteins (CAT,

$\mathrm{Cu} / \mathrm{Zn}-\mathrm{SOD}, \mathrm{Mn}-\mathrm{SOD})$, decrease in

photosynthesis and Calvin

cycle-related proteins (RubisCO; PSI

subunit VII PsaC)

Genotypic differences:

COR410-higher increase in T than $\mathrm{S}$
Common wheat cv. Nesser (T), Opata M85 (S)-root $21^{\circ} \mathrm{C} ; 40 \%$ humidity-combined effect of drought and ABA $(100 \mu \mathrm{M})$
nanoLC-MS/MS ITRAQ

1656 identified proteins

805 ABA-responsive proteins: LEA

protein phosphatases PP2C;

Genotypic differences: HSP70, HSP90; 14-3-3, G-proteins;

V-ATPase-higher in T; $\beta$-expansin, porins-higher in $\mathrm{S}$

\section{6 identified proteins}

Caruso et al., 2009

Up: carbonic anhydrase, RubisCO LSU

Down: RubisCO SSU, Calvin cycle enzymes (ALDO, PRK); ATP synthase CF1 $\alpha$; plastidic GS2a,b,c

75 identified proteins, 11 candidates for drought tolerance

Genotypic differences: TPI, ATP synthase CF1 (efficient carbohydrate metabolism and ATP production)-higher in $\mathrm{T}$; $\beta$-1,3-glucanase, $\beta$-1,4-glucanase, XET (cell wall remodeling for osmotic adjustment and energy source); methionine synthase-higher in S 
Table 1 | Continued

\section{Plant material}

Treatment

Methods

\section{Major differentially- abundant proteins \\ (DAP)}

\section{Barley cv. Basrah (T) and} Golden Promise (S)-leaf, root

$\begin{array}{ll}7 \text { days without } & 10 \mathrm{mM} \text { PBS, } \\ \text { watering } & \text { TCA-acetone; 2D-DIGE } \\ \text { Control: } 80 \% \text { RWC; } & \text { MALDI-TOF }\end{array}$

Drought: $70 \%$ RWC

(T), $60 \%$ RWC (S)
Identified proteins: 24 (leaf), 45 (root)

Up: ABA-induced protein $\mathrm{r} 40 \mathrm{c} 1$, small

G-protein Rab2, Myb-like protein,

14-3-3 protein

Down: GST, GPX

Genotypic differences: Enhanced regulation of ROS (APX, CAT, LOX, class III POX) and protein folding in $T$ than in $\mathrm{S}$

\section{Barley-8 Egyptian} accessions, 2 selected for proteome analysis 15141 (T), 15163 (S)-leaf $24^{\circ} \mathrm{C} ; 70 \%$ FWC (control); 5 days at 10 $\%$ FWC (stress)
TCA/acetone; 2D-DIGE MALDI-TOF
Up: PDI, Hsp90, Hsp100 (Clp protease), chloroplastic ATP synthase CF1 $\alpha$; Genotypic differences: PPDK, Hsp70, zinc metalloprotease-higher in $\mathrm{T}$ than S; proteins involved in osmolyte biosynthesis (betaine aldehyde dehydrogenase, methionine synthase, SUS1)-higher in $S$ than $T$

\section{Barley cv. Golden} Promise-leaf

$\begin{array}{ll}\text { Ca } 100 \% \text { FWC } & \text { TCA/acetone; 2DE } \\ \text { (control); } 25 \% \text { FWC } & \text { MALDI-TOF/TOF } \\ \text { (stress)-28 } & \\ \text { days-combined } & \\ \text { effect of drought and } & \\ \text { Piriformospora indica } & \end{array}$

Piriformospora indica

\section{5 identified proteins}

Up: RubisCO activase A, RubisCO

SSU, CCOMT

Down: PRK, ACP

Effect of $P$. indica: RubisCO SSU, PSI

Fe-S center, chl $a / b$ binding protein; CCOMT, APX, 30S ribosomal protein 3, V-ATPase, 2-Cys Prx-higher in inoculated than control plants under stress

\section{OSMOTIC STRESS (PEG-6000)}

Common wheat cv. Yumai 34-leaf
Hoagland solution, $15 \%$ PEG-6000 (3 days); 0.5 mM SA pretreatment (3 days)

Common wheat-spring wheats Abbondanza ( $\mathrm{T})$, Qingchun 38 (S)-leaf

PEG-6000 (-1 MPa;

$72 \mathrm{~h})$, recovery $(24 \mathrm{~h})$
TCA/acetone; 2DE MALDI-TOF/TOF
82 (76 identified proteins), of which 35 Kang et al., 2012

SA-responsive proteins

Up: 14-3-3; APX, GST,

SA-responsive proteins: GS1c, GST1, PDI; ATP synthase CF1 $\alpha, \beta$

38 (35 identified proteins) $\quad$ Ye et al., 2013
Up: GAPDH B; 26S proteasome,
V-ATPase A

\section{V-ATPase A}

Down: RubisCO LSU and SSU,

GAPDH, TPI, AGPase (starch

biosynthesis)

Genotypic differences: Less

PEG-affected proteins in $T$ than $S$

\begin{tabular}{lll}
\hline Common wheat cv. & Hoagland solution, & TCA/acetone/phenol; \\
Hanxuan $10(\mathrm{~T})$ and & $20 \% \mathrm{PEG}-6000$ & $\begin{array}{l}\text { phosphopeptide } \\
\text { enrichment via } \mathrm{TiO}_{2}\end{array}$ \\
$\begin{array}{l}\text { Ningchun } 47(\mathrm{t}) \text {-seedling } \\
\text { leaf }\end{array}$ & $(-075 \mathrm{MPa})$ for 48 h & $\begin{array}{l}\text { microcolumns; } \\
\text { LC-MS/MS }\end{array}$
\end{tabular}

$173(\mathrm{~T})$ and 251 (t) phosphoproteins identified

Phosphoproteins identified: signaling (SnRK2 kinase, protein phosphatase 2C, CDPK, calmodulin 2-2); transport (AQP, MSSP2; H+-ATPase); LEA proteins (WCOR719, WCOR825, WRAB17)
Wendelboe-Nelson and Morris, 2012
References

Ashoub et al., 2013 
Table 1 | Continued

\begin{tabular}{lll}
\hline Plant material & Treatment
\end{tabular}

Major differentiallyabundant proteins

(DAP)

\section{SALINITY}

Common wheat

(T. aestivum) Jinan 177

(S), T. aestivum $x$

Thinopyrum ponticum

Shanrong 3 (T)-seedling

root

\section{1/2Hoagland solution, TCA/acetone; 2DE}

$200 \mathrm{mM} \mathrm{NaCl}(24 \mathrm{~h}) \quad$ MALDI-TOF,

MALDI-TOF/TOF
114 (110 identified-49 salt-responsive, Wang et al., 2008

34 genotypic differences)

Up: 14-3-3;

Down: tubulin $\alpha-3$

Genotypic differences: DEAD-box

RNA helicase, DWARF3 (GA

biosynthesis), elF5A2, V-ATPase

subunit $\mathrm{E}$-higher in T; G-protein $\beta$

subunit, ethylene receptor

ETR1-higher in S
Common wheat cv.

Calingiri, Janz (S),

Wyalkatchem (T)-shoot

mitochondrial fraction

$\begin{array}{ll}200 \mathrm{mM} \mathrm{NaCl} & \text { Isolation: PVP gradient; } \\ \text { (increase per } 50 \mathrm{mM} & \text { acetone extraction; } \\ \mathrm{NaCl} / \text { days) } & \text { 2D-DIGE LC-MS/MS }\end{array}$

192 DAP (68 identified)

Up: AOX, Mn-SOD, VDAC

Down: CS, NDPK, outer mitochndrial

membrane porin

Genotypic differences: AOX,

Mn-SOD-higher in T

\begin{tabular}{lll}
\hline Common wheat cv. & $150 \mathrm{mM} \mathrm{NaCl}$ & Isolation: Percoll \\
Keumgang-leaf & $(1,2,3$ days $)$ & gradient; TCA/acetone;
\end{tabular}

chloroplast fraction

100 DAP (65 identified)

Up: RubisCO, GAPDH, GDH, PDX1.2

and PDX1.3

Down: ATP synthase $\alpha, \beta, \gamma$; V-type

proton ATPase
References

Jacoby et al., 2010
Kamal et al., 2012 2DE LTQ-FTICR-MS
55 root, 15 shoot differentially abundant proteins

Organ-specific differences: aspartate aminotransferase, GDH (up in shoot, down in root)

Genotypic differences: Mn-SOD,

$\mathrm{MDH}$, aconitase, SHMT, $\beta$-CAS-higher in $\mathrm{T}$
Durum wheat (T. durum) cv. Ofanto -leaf

$\begin{array}{ll}100 \mathrm{mM} \mathrm{NaCl}(2 & \text { TCA/acetone 2DE } \\ \text { days) } & \text { MALDI-TOF }\end{array}$

38 identified proteins

Up (28): TPI; CPN60- $\beta$, RubisCO

activase, carbonic anhydrase;

osmolyte biosynthesis-related

enzymes (glycine dehydrogenase,

SAMS); COR; Cu/Zn-SOD

Down (10): ALDO, PGK, RubisCO

SSU, OEE1 precursor, $\beta$-glucosidase,

ATP synthase CF1 $\alpha$

\begin{tabular}{lll}
\hline Durum wheat cv. & $250 \mathrm{mM} \mathrm{NaCl}$ & $\mathrm{KCl}(100 \mathrm{mM})$, \\
Waha-seed embryo and & $(42 \mathrm{~h})-\mathrm{AsA}$ priming & acetone/nanoHPLC-MS \\
surrounding tissue & $(0.5 \mathrm{mM})$ &
\end{tabular}

697 identified proteins-proteins involved in energy metabolism, protein metabolism, disease/defense, protein destination, storage-a positive effect of AsA priming on mitigation of salinity stress

\begin{tabular}{ll}
\hline Barley cv. OUK305 (T), & $\begin{array}{l}200 \mathrm{mM} \mathrm{NaCl}(5 \\
\text { days) }\end{array}$
\end{tabular}

\author{
$40 \mathrm{mM}$ Tris, $8 \mathrm{M}$ urea, \\ 4\% CHAPS, $0.2 \%$ \\ Bio-Lyte; 2DE
} nanoLC-ESI-MS/MS

$\begin{array}{ll}6 \text { differentially abundant proteins } & \text { Sugimoto and } \\ \text { CCOMT, DHAR, GST (2 spots), POX, } & \text { Takeda, } 2009 \\ \text { PR10-higher in T than S } & \end{array}$

Fercha et al., 2013, 2014

Jacoby et al., 2013

Caruso et al., 2008 
Table 1 | Continued

\begin{tabular}{|c|c|c|c|c|}
\hline Plant material & Treatment & Methods & $\begin{array}{l}\text { Major differentially- } \\
\text { abundant proteins } \\
\text { (DAP) }\end{array}$ & References \\
\hline $\begin{array}{l}\text { Barley cv. Afzal (T), L-527 } \\
\text { (S)-leaf }\end{array}$ & $\begin{array}{l}300 \mathrm{mM} \mathrm{NaCl} \\
\text { (increase per } 50 \mathrm{mM} \\
\mathrm{NaCl} / \text { days) } 24 \mathrm{~h}\end{array}$ & $\begin{array}{l}\text { TCA/acetone; 2DE } \\
\text { MALDI-TOF/TOF }\end{array}$ & $\begin{array}{l}117 \text { DAP (22 identified proteins) } \\
\text { Up:, PC, OEE2, PSI subunit VII (PsaC), } \\
\text { PRK; 2-Cys Prx, Trx, GST, SOD; TPI, } \\
\text { FBP ALDO-higher in T than S }\end{array}$ & Rasoulnia et al., 2011 \\
\hline $\begin{array}{l}\text { Barley cv. Morex (T), } \\
\text { Steptoe (S)-root }\end{array}$ & $\begin{array}{l}100,150 \mathrm{mM} \mathrm{NaCl} \\
(0,1,4,7,10 \text { days })\end{array}$ & $\begin{array}{l}\text { TCA/acetone; 2DE } \\
\text { MALDI-TOF; } \\
\text { nanoLC-ESI-Q-TOF } \\
\text { MS/MS }\end{array}$ & $\begin{array}{l}91 \text { DAP (74 identified proteins) } \\
\text { Genotypic differences: GLP3-7, } \\
\text { GLP12, } \beta \text {-1,3-glucanase, ATP synthase } \\
\text { CF1 } \beta \text {-higher in T; GLP5a, } \\
\text { PR17-higher in S }\end{array}$ & Witzel et al., 2014 \\
\hline $\begin{array}{l}\text { Drought and heat } \\
\text { Barley-Syrian landrace } \\
\text { Arta (T), Australian cv. } \\
\text { Keel (T)-leaf (heading } \\
\text { stage) }\end{array}$ & $\begin{array}{l}\text { Drought: } 50 \% \text { FWC } \\
\text { (control), } 15 \% \text { FWC } \\
\text { (stress) for } 3 \text { days } \\
\text { Heat: } 36^{\circ} \mathrm{C}(4 \mathrm{~h})\end{array}$ & $\begin{array}{l}\text { TCA/acetone; 2DE, } \\
\text { 2D-DIGE } \\
\text { MALDI-TOF/TOF }\end{array}$ & $\begin{array}{l}99 \text { DAP } \\
\text { Heat-up: FBP ALDO, chaperones, } \\
\text { proteases, eEF-G, elF4A, RubisCO } \\
\text { activase B } \\
\text { Genotypic differences (14 proteins): } \\
\text { photosynthesis-related proteins } \\
\text { (LHCII type III Lhcb3, OEE1 PsbO, } \\
\text { RubisCO activase B)-higher in Keel } \\
\text { than Arta }\end{array}$ & Rollins et al., 2013 \\
\hline $\begin{array}{l}\text { Drought or waterlogging } \\
\text { and cold-winter common } \\
\text { wheat cv. Yannong } \\
\text { 19-leaf }\end{array}$ & $\begin{array}{l}\text { Drought }+\mathrm{LT}: 35 \% \\
\text { FWC (7 days) } \\
\text { Waterlogging }+\mathrm{LT} \\
\text { (7 days) }\end{array}$ & $\begin{array}{l}\text { TCA/acetone; 2DE } \\
\text { MALDI-TOF/TOF }\end{array}$ & $\begin{array}{l}32 \text { identified proteins } \\
\text { Up: DHAR, GR; Hsp70; } \\
\text { Down: C metabolism-related proteins } \\
\text { (glycolysis, TCA, Krebs cycle), } \\
\text { RubisCO activase A, ATP synthase } \\
\text { CF1 } \alpha, \beta\end{array}$ & Li et al., 2014 \\
\hline
\end{tabular}


Table 1 | Continued

\begin{tabular}{|c|c|c|c|c|}
\hline Plant material & Treatment & Methods & $\begin{array}{l}\text { Major differentially- } \\
\text { abundant proteins } \\
\text { (DAP) }\end{array}$ & References \\
\hline
\end{tabular}

\section{IMBALANCES IN MINERAL NUTRIENTS}

\section{Boron}

Barley Clipper (S) $\times$

Sahara (T) DH lines-leaf, $\quad 5 \mathrm{mM} \mathrm{H}_{3} \mathrm{BO}_{3}$ (T) for

PBS pH 7.5,

TCA/acetone;

14 days 2D-nanoLC-MS/MS

root

iTRAQ

(n)

138 (leaf), 341 (root) identified
proteins
Up: IDS2, IDS3, methyl-thioribose
kinase
Leaf: PRK, PGK, PGM, ENO, PC,
RubisCO activase, eEF1- $\alpha, \beta, \gamma ;$ eEF-G,
eEF-Tu; TLP; Cu/Zn-SOD; 50S
ribosomal protein L3; 60S ribosomal
protein L1
Root: CCOMT, class III POX, chitinase,
26S proteasome, $\beta-1,3-$ glucanase;
ATP synthase CF1 $\beta$, IDS2, IDS3;
Hsp70; Hsc70; 40S ribosomal protein
S5
98 DAP [93 identified proteins-43
(leaf), 49 (root)]
36 Cu-responsive proteins
Leaf: $14-3-3 ;$ MDH, TPI; PDI; V-ATPase
A; ATP synthase CF1 $\alpha$; carbonic
anhydrase, RubisCO activase, PSI
subunit VII (PsaC);
Root: $14-3-3$, translationally-controlled 2013
tumor protein; Hsp70, APX, GST,
Cu/Zn-SOD, PR10; TPI, ATP synthase
CF1 $\alpha ;$ actin 1, tubulin

$\begin{array}{llll}\text { Nitrogen } & & \\ \text { Common wheat cv. } & 2,8,20 \mathrm{mg} \mathrm{N} / \text { plant/d } & \text { TCA/acetone; 2DE } & \text { 76 DAP (14 identified proteins) } \\ \text { Arche, Récital-leaf } & \text { for } 60 \text { days } & \text { LC-MS/MS } & \text { FBP ALDO, PGK, PGM, ENO2, MDH; } \\ & & \text { RubisCO activase A, OEE1 (PsbO); } \\ & & \text { 2-Cys Prx }\end{array}$

\section{Copper}

Common wheat cv. $\quad 100 \mu \mathrm{M} \mathrm{CuSO}_{4} \quad$ TCA/acetone; 2DE

Yumai 34-leaf, root

(3 days)

MS/MS

\section{PATHOGENS}

Fusarium culmorum and $\boldsymbol{F}$ graminearum (teleomorph Gibberella zeae)

Barley cv. Scarlett

(S)-young spikelet

artificial inoculation

F. Graminearum-3

Acetone; 2DE

days

MALDI-TOF

artificial inoculation

$5 \mathrm{mM}$ Tris- $\mathrm{HCl} \mathrm{pH} 7.5$

Barley cv. Scarlett

(S)-mature grain

F. Graminearum-

$72 \mathrm{~h}$;

$15,100 \mathrm{~kg} \mathrm{ha}^{-1} \mathrm{~N}$ 2DE MS/MS (water soluble proteins),

2-Cys Prx

51 DAP (50 identified) Yang et al., 2010a

Up: PR proteins (PR-1,2,3,5,9,15);

proteolytic fragments of $\beta$-amylase induced by pathogen

Up: 80 proteins (serpin, protease

Yang et al., 2010b

inhibitors $\mathrm{Cl}-1 \mathrm{~A}, \mathrm{Cl}-1 \mathrm{~B})$

Down: 108 proteins (albumins)

65 proteolytic fragments (albumins,

serpin, protease inhibitors)

9 proteins of $F$ graminearum

(peptidyl-prolyl cis-trans isomerase,

Cu/Zn-SOD, L-xylulose reductase)

Positive effect of increased $\mathrm{N}$ on plant

resistance

Naked barley (Hordeum

vulgare ssp.

$1.2 \mathrm{mg} / \mathrm{kg}$ DON

(artificial inoculation

F. culmorum and

F. graminearum)
$50 \mathrm{mM}$ Tris- $\mathrm{HCl} \mathrm{pH} \mathrm{7.4;}$

NEPHGE 2-DE

MALDI-TOF

nanoLC-MS/MS
11 identified proteins

Up: DNA-dependent

RNA-polymerase; Dof zinc-finger

protein, NBS-LRR (transcription

regulation); serpin (3 spots; serine
Eggert and Pawelzik, 2011 
Table 1 | Continued

\begin{tabular}{|c|c|c|c|c|}
\hline Plant material & Treatment & Methods & $\begin{array}{l}\text { Major differentially- } \\
\text { abundant proteins } \\
\text { (DAP) }\end{array}$ & References \\
\hline
\end{tabular}

protease inhibitor);

Down: ADP-glucose

pyrophosphorylase

\begin{tabular}{lll}
\hline Emmer (Triticum & $10 \mathrm{mg} / \mathrm{kg} \mathrm{DON}$ & TCA/acetone; 2DE \\
dicoccum)-mature grain & (artificial inoculation) & nanoLC-MS/MS
\end{tabular}

10 identified proteins

Eggert et al., 2011

Up: serpin (serine protease inhibitor), TLP; $\beta$-amylase, globulin Down: POX, Prx; glycosyltransferase; chitinase; $\alpha$-gliadin

\title{
Puccinia triticina
}

Common wheat cv. Thatcher (S), NIL ThatcherLr1 (T)-leaf

\author{
3, 6, 9 days after TCA/acetone; 2DE \\ artificial infection $\quad$ qTOF-MS/MS
}

\author{
32 identified proteins (S); T showed \\ no reproducible response \\ Rampitsch et al., \\ 2006 \\ Up: eEF1- $\beta$, elF5A2, 20 S proteasome \\ subunit $\alpha-1$, ribosomal protein P0; TPI; \\ dihydrolipoamide acetyl transferase; \\ $\alpha$-tubulin; Hsp70, CPN60; ATP \\ synthase CF1 $\beta$; peptidyl-prolyl \\ cis-trans isomerase
}

\section{Septoria tritici (teleomorph Mycosphaerella graminicola)}

Common wheat cv. Sevin

(S), Stakado (T)-leaf

\section{$3,7,11$ days after} artificial infection

\author{
Phenol extraction; \\ phosphoprotein \\ separation: Poros Oligo \\ R3 micro-column; \\ LC-MS/MS
}

\author{
Plant: Phosphoproteins (70 in T, 60 in Yang et al., 2013 \\ S)-signaling (CDPK, MAPK); transport \\ (PIP ATPase)-higher in $\mathrm{T}$ than $\mathrm{S}$ \\ Pathogen: 31 proteins, 5 \\ phosphoproteins (G-proteins, 14-3-3; \\ Ras GTPase; ABC transporter)
}

\begin{abstract}
Abbreviations: 2Cys-Prx, 2-cysteine peroxiredoxin; 2DE, two-dimensional electrophoresis; 2D-DIGE, two-dimensional differential in-gel electrophoresis; $\beta$-CAS, $\beta$ cyanoalanine synthase; $A B A$, abscisic acid; $A C P$, acyl carrier protein; AGPase, ADP glucose pyrophosphorylase; $A O X$, alternative oxidase; $A P X$, ascorbate peroxidase; $A Q P$, aquaporin; AsA, ascorbic acid; CCOMT, caffeoyl-coenzyme A O-methyltransferase; COR, Cold-regulated (protein); CPN, chaperonin; CS, cysteine synthase; CDPK, calcium-dependent protein kinase; DAP, differentially abundant proteins; DH, double haploid (line); DHAR, dehydroascorbate reductase; DON, deoxynivalenol; ENO, enolase; FBP ALDO, fructose-1,6-bisphosphate aldolase; FWC, field water capacity; GAPDH, glyceraldehyde-3-phosphate dehydrogenase; GAPDH B, glyceraldehyde-3-phosphate dehydrogenase B form; GDH, glutamate dehydrogenase; GLP, germin-like protein; GPX, glutathione peroxidase; GS, glutamine synthetase; GST, glutathione S-transferase; HPLC, high performance liquid chromatography; Hsc70, heat shock cognate protein 70; iTRAQ, isobaric tag for relative and absolute quantification; LC, liquid chromatography; LEA, Late embryogenesis-abundant (protein); LOX, lipoxygenase; LTQ-FTICR, linear quadruple trap-Fourier transform ion cyclotron resonance; MALDI-TOF/TOF, matrix-assisted laser desorption ionization time-of-flight/time-of-flight (spectrometry); MAPK, mitogen-activated protein kinase; MDAR, monodehydroascorbate reductase; $M D H$, malate dehydrogenase; $M S$, mass spectrometry; MSSP2, monosaccharide sensing protein 2; NBS$L R R$, nucleotide-binding site leucine-rich repeat protein; NEPHGE, non-equilibrium pH gel electrophoresis; NDPK, nucleoside diphosphate kinase; NIL, near-isogenic line; OEE, oxygen evolving enhancer (protein); PBS, phosphate buffer saline; $P C$, plastocyanin; $P D I$, protein disulfide isomerase; $P D X$, pyridoxal biosynthesis protein; PEG, polyethylene glycol; PGK, phosphoglycerokinase; PGM, phosphoglyceromutase; POX, peroxidase; PPDK, pyruvate phosphate dikinase; PRK, phosphoribulokinase; Prx, peroxiredoxin; PS, photosystem; PVP, polyvinyl pyrrolidone; qTOF, quadrupole time-of-flight; RubisCO, ribulose-1,5-bisphosphate carboxylase/oxygenase; RubisCO LSU, RubisCO large subunit; RubisCO SSU, RubisCO small subunit; RWC, relative water content; S, sensitive (genotype); SA, salicylic acid; SHMT, serine hydroxymethyltransferase; SnRK, sucrose non-fermenting-related protein kinase; SOD, superoxide dismutase; SUS1, sucrose synthase 1; T, tolerant (genotype); $t$, genotype less tolerant than T; TCA, trichloroacetic acid; TLP, thaumatin-like protein; TPI, triose phosphate isomerase; Trx, thioredoxin; V-ATPase, vacuolar ATPase; VDAC, voltage-dependent anion channel; WCS, Wheat Cold-specific (protein); WRAB, Wheat responsive-to-ABA (protein); XET, xyloglucan endo-transglycosylase.
\end{abstract}

differentially-tolerant genotypes may help researchers to identify key proteins underlying the differences in stress tolerance.

The aim of this minireview is to summarize the major results obtained by proteomic studies in temperate cereal crops wheat and barley studied under abiotic and biotic stresses. Proteins affected by differential stress factors and proteins revealing a differential response between differentially-tolerant wheat and barley genotypes are discussed in a greater detail. Possibilities of utilization of proteins revealing a differential stress response between tolerant and sensitive genotypes as protein markers in breeding programs aimed at improvement of stress tolerance are suggested.

\section{COMMON FEATURES OF STRESS RESPONSE AT PROTEOME LEVEL}

Plant stress response is a dynamic process aimed at an enhancement of plant stress tolerance and an establishment of a novel homeostasis between plant and environment (Figure 1). Several 


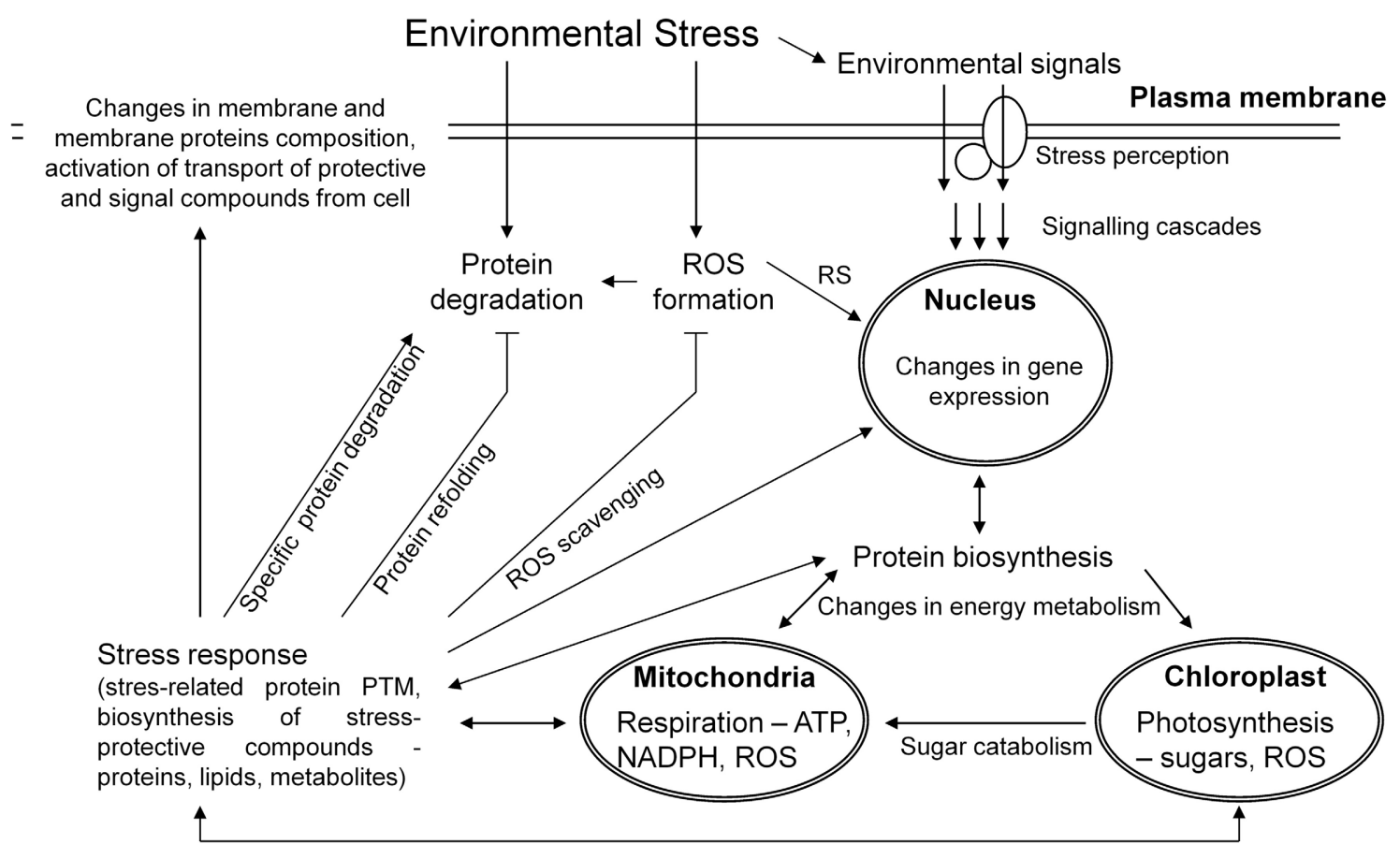

FIGURE 1|A simplified scheme of plant cell response to an external stress stimulus leading to an activation of signaling cascades, changes in gene expression, activation of protein biosynthesis and degradation, profound changes in energy metabolism leading to an enhanced ATP biosynthesis and ROS production in chloroplasts and mitochondria resulting in ROS induced signaling (RS). Changes in protein biosynthesis lead to an enhanced production of both protein and non-protein (metabolite) stress protective compounds including ROS scavenging enzymes and metabolites, which participate in an active plant stress acclimation response including a feedback regulation of stress-induced signaling, gene, and protein expression mechanisms. phases of plant stress response could be distinguished including an alarm phase, an acclimation phase, a resistance phase, an exhaustion phase when stress is too severe or lasts too long, and a recovery phase after a cessation of the given stress factor (Levitt, 1980; Larcher, 2003; Kosová et al., 2011). At proteome level, profound alterations in protein relative abundance were found between stressed and control plants as well as between differential genotypes (Table 1). During an alarm phase, stress induces profound alterations in proteins involved in cell signaling although these proteins are detected scarcely on 2DE gels due to their low abundance. An increase in 14-3-3 proteins as well as translationally controlled tumor protein homologs was detected in copperand water-stressed wheat (Kang et al., 2012; Ghabooli et al., 2013; Li et al., 2013; Alvarez et al., 2014) and barley (Wendelboe-Nelson and Morris, 2012) and genotype-specific responses of $\beta$ subunit of heterotrimeric $\mathrm{G}$ protein were found in salt-stressed wheat (Peng et al., 2009). Phosphorylation plays an important role in abiotic and biotic stress responses as shown on several kinases (calciumdependent protein kinases CDPK, mitogen-activated protein kinases MAPK, sucrose non-fermenting-related kinases SnRK2), phosphatases (PP2C) and other signaling proteins (calmodulin 2-2) regulation (Yang et al., 2013; Zhang et al., 2014).

Stress acclimation represents an adaptive process aimed at an enhancement of plant stress tolerance. An active stress acclimation requires relatively high energy costs as indicated by profound alterations in energy metabolism. Practically all stresses induce an increased relative abundance of enzymes of carbohydrate catabolism such as glycolysis (glyceraldehyde-3-phosphate dehydrogenase GAPDH, triosephosphate isomerase TPI, enolase ENO), Krebs cycle (mitochondrial $\mathrm{NAD}^{+}$-dependent malate dehydrogenase (MDH; Vítámvás et al., 2012), aconitase (Jacoby et al., 2010, 2013; Budak et al., 2013) and components of mitochondrial ATP-synthase, namely $\beta$ subunit of CF1 complex (Bahrman et al., 2004; Patterson et al., 2007; Vítámvás et al., 2012; Budak et al., 2013; Kosová et al., 2013b; Rollins et al., 2013; Xu et al., 2013) indicating an enhanced demand for energy. Regarding photosynthesis, an increase or a decrease in several photosynthetic proteins (proteins involved in primary photosynthetic reactions, carbon fixation, and Calvin cycle) have been observed depending on the severity of stress (Caruso et al., 2008, 2009; Ye et al., 2013). A downregulation of photosynthesis reactions under severe stress is reflected at proteome level by a decrease in D1 and D2 proteins in photosystem II reaction center (RC PSII), proteins of oxygen evolving complex (OEC), a decrease in chlorophyll $a-b$ binding proteins in both photosystem (PS) I and II, a decrease in Fe-S complex in PSI, a downregulation of RubisCO and key Calvin cycle enzymes phosphoglycerate kinase and phosphoribulokinase in cold- and waterlogging-treated winter wheat (Li et al., 2014), salt-treated durum wheat (Caruso et al., 2008) and in drought-treated barley (Ghabooli et al., 2013) while an increase in OEC protein OEE2 was found in salt-treated barley (Rasoulnia et al., 2011; Fatehi et al., 2012). In addition, an 
increase in proteins with stimulating and protective functions such as RubisCO activase A (Bahrman et al., 2004; Caruso et al., 2008, 2009; Fatehi et al., 2012; Budak et al., 2013), a thermostable RubisCO activase B (Rollins et al., 2013), carbonic anhydrase (Caruso et al., 2008) and RubisCO large and small subunit binding proteins $\mathrm{CPN} 60-\alpha$ and $\mathrm{CPN} 60-\beta$ was observed under various stresses (Caruso et al., 2008; Sarhadi et al., 2010; Kang et al., 2012; Budak et al., 2013; Kosová et al., 2013b; Xu et al., 2013).

An increased demand on energy under stress acclimation corresponds with a decreased abundance of enzymes (fructokinase2, sucrose synthase-1) involved in biosynthesis of energy-rich compounds such as starch and a decrease in storage proteins (11S seed storage protein 2-like, legumin-like protein; Vítámvás et al., 2012; Kosová et al., 2013b).

Stress acclimation also reveals an enhanced demand on protein metabolism including both protein biosynthesis and degradation. Changes in the levels of eukaryotic translation initiation factors eIF3 subunit I, eIF5A2 (Kosová et al., 2013b), eIF4A (Rollins et al., 2013) and elongation factor eEF1- $\alpha$ (Budak et al., 2013), several ribosomal proteins, e.g., 60S proteins P0, P2A, L3, L38 (Fercha et al., 2014), or chloroplastic ribosomal proteins 30S-3, 50S-L12 (Ghabooli et al., 2013; Gharechahi et al., 2014), as well as in proteasome subunits such as $20 \mathrm{~S}$ proteasome subunit $\alpha$ type 1 and 6 (Rampitsch et al., 2006; Rinalducci et al., 2011a; Fercha et al., 2013; Ghabooli et al., 2013) and proteins of ubiquitin pathway involved in proteasome targeting such as ubiquitin conjugating enzyme E2 variant IC like (Kosová et al., 2013b) were reported indicating an enhanced protein turnover during stress acclimation.

Stress represents an enhanced risk of protein damage due to imbalances in cellular homeostasis. Therefore, increased abundances of several proteins with chaperone and other protective functions have been reported. Extreme temperatures, but also drought, pathogens, and other stresses cause an enhanced risk of protein misfolding and they are thus associated with an enhanced accumulation of chaperones from HSP superfamily, namely HSP70 (Rampitsch et al., 2006; Li et al., 2013; Rollins et al., 2013), HSP100 (Clp protease; Ashoub et al., 2013), and small HSPs (Skylas et al., 2002; Majoul et al., 2004; Hajheidari et al., 2007), but also others such as chopper chaperone (Vítámvás et al., 2012; Hlaváčková et al., 2013), serpins (Yang et al., 2010b; Fercha et al., 2013, 2014), and protein disulfide isomerase (Hajheidari et al., 2007; Vítámvás et al., 2012; Li et al., 2013). However, a decrease in HSP90 was reported under cold (Vítámvás et al., 2012). Disorders in cellular metabolism under stress lead to an enhanced risk of oxidative damage. At proteome level, an increased abundance of several reactive oxygen species (ROS) scavenging enzymes was found practically under each kind of stress (Hajheidari et al., 2007; Ford et al., 2011). Plants try to reduce a risk of ROS formation by several ways. The major one represents a downregulation of photosynthesis reactions which is associated with a decrease in D1 and D2 proteins in photosystem II reaction center (RC PSII), proteins of OEC, RubisCO small subunit and key Calvin cycle enzymes phosphoglycerate kinase, phosphoribulokinase and transketolase (Caruso et al., 2008; Ford et al., 2011; Ashoub et al., 2013). Other indirect ways how to reduce ROS lie in a reduced uptake of metal ions, especially iron, which can act as catalyzers of ROS formation. A reduced level of protein IDI2 and dioxygenases IDS2, IDS3 involved in iron uptake and phytosiderophore biosynthesis was found by Witzel et al. (2009) in salt-treated barley roots while an increased level of these enzymes was found by Patterson et al. (2007) in barley grown under elevated boron.

Several stresses including drought, heat, salinity, cold, but also mechanical wounding, induce an enhanced accumulation of proteins belonging to LEA superfamily. Late embryogenesisabundant (LEA) superfamily includes at least five subclasses, the most important being LEA-II (dehydrins) and LEA-III proteins whose transcript and protein levels, and also phosphorylation level, have been reported to correlate with wheat and barley tolerance to low temperatures (Crosatti et al., 1995; Vágújfalvi et al., 2000, 2003; Vítámvás et al., 2007; Kosová et al., 2008c, 2013c; Sarhadi et al., 2010), drought (Labhilili et al., 1995; Brini et al., 2007) and other stresses.

Several stresses, especially biotic ones, are associated with an induction of protective proteins from $\mathrm{PR}$ superfamily. Pathogenesis-related (PR) proteins encompass 16 groups involved in defense against microbial and fungal pathogens (Edreva, 2005). Many of $\mathrm{PR}$ proteins can resist acidic $\mathrm{pH}$, they reveal enzymatic activities aimed at modifications of cell wall, and ROS scavenging functions (some germins and germin-like proteins reveal manganese superoxide dismutase (Mn-SOD) and oxalate oxidase activities). An enhanced abundance of several PR proteins was reported not only in cereals exposed to fungal pathogens such as Fusarium (class-II chitinase, $\beta$-amylase, thaumatin-like protein, PR9-peroxidase; Yang et al., 2010a,b; Eggert and Pawelzik, 2011; Eggert et al., 2011), but also under abiotic stresses such as cold ( $\beta$-1,3-glucanase, chitinase, PR4, thaumatin-like protein; Sarhadi et al., 2010; Kosová et al., 2013b; Gharechahi et al., 2014), salinity (germin-like protein, PR10; Fatehi et al., 2012; Kamal et al., 2012; Witzel et al., 2014), and others.

Stresses also affect other aspects of cellular metabolism. An increased abundance of methionine synthase catalyzing formation of methionine or S-adenosylmethionine synthase (SAMS) catalyzing formation of S-adenosylmethionine (SAM) has been reported (Bahrman et al., 2004; Patterson et al., 2007; Witzel et al., 2009; Vítámvás et al., 2012; Kosová et al., 2013b; Xu et al., 2013). SAM represents not only a universal methyl donor in regulation of DNA heterochromatin formation and gene expression, but it is also a precursor of several stress-related metabolites as glycine betaine, polyamines, hydroxymugineic acids (phytosiderophore precursors; Mori and Nishizawa, 1987) and ethylene. Alterations in glutamine synthetase (GS) have been reported under drought (Ford et al., 2011; Kang et al., 2012) and cold (Hlaváčková et al., 2013 ) indicating an important role of nitrogen assimilation and proline biosynthesis in stress acclimation.

Stress affects cellular transport and membrane properties. An enhanced need for ion transport and thus an associated increase in plasma membrane and tonoplast ion transporters such as VATPase has been reported not only under salinity (Peng et al., 2009), but also under other stresses such as drought (Ghabooli et al., 2013), heat (Majoul et al., 2004) and osmotic stress (Ye et al., 2013; Zhang et al., 2014). Differential phosphorylation of several transport proteins such as aquaporins, $\mathrm{H}^{+}$-ATPase or 
monosaccharide sensing protein 2, was also reported in response to stress (Zhang et al., 2014). The effect of several stresses on cell wall remodeling is indicated by alterations in several enzymes involved in lignin metabolism such as caffeoyl-coenzyme A O-methyltransferase CCOMT indicating an important role of cell wall in plant stress response (Sugimoto and Takeda, 2009; Ghabooli et al., 2013).

Long-term and regularly occurring stress factors such as cold during winter also affect plant development. At proteome level, significant changes in the level of small glycine-rich RNA-binding proteins (sGRPs) and in lectins, glycoproteins involved in saccharide signaling, were found in wheat (Rinalducci et al., 2011b; Kosová et al., 2013b). Ricin B lectin 2 was reported to be induced by cold in crowns of both winter barley (Hlaváčková et al., 2013) and winter wheat (Kosová et al., 2013b). Lectin VER2 was reported to accumulate in winter wheat shoot apex until vernalization (Yong et al., 2003; Rinalducci et al., 2011b). Differences in sGRPs and VER2 levels between spring and winter wheat growth habits indicate a differential response to cold within wheat germplasm (Kosová et al., 2013b).

\section{PROTEINS REVEALING A DIFFERENTIAL RESPONSE BETWEEN STRESS-TOLERANT AND STRESS-SENSITIVE GENOTYPES}

A differential ability of various wheat and barley genotypes to cope with several stresses is reflected also at protein level. Stresstolerant genotypes do not suffer from a disruption of energy metabolism when exposed to moderate stress levels; moreover, when exposed to stress, they can increase an abundance of key enzymes of energy metabolism to increase ATP production as indicated by a differential response observed in several photosynthesis-related proteins (RubisCO subunits, RubisCO activase), ROS scavenging enzymes as well as respiration (Krebs cycle) enzymes. Quantitative differences in Krebs cycle enzymes such as mitochondrial $\mathrm{NAD}^{+}$-dependent MDH between two differentially frost-tolerant winter wheats (Vítámvás et al., 2012), in aconitase (Budak et al., 2013), thioredoxin $h$ and glutathione-Stransferase (GST; Hajheidari et al., 2007; Sarhadi et al., 2010), lipoxygenase 1 and 2 (Alvarez et al., 2014) between differentially drought-tolerant wheats; in $\mathrm{Cu} / \mathrm{Zn}-\mathrm{SOD}, \mathrm{Mn}-\mathrm{SOD}$ (Ford et al., 2011; Xu et al., 2013), glyoxysomal MDH (gMDH; Ashoub et al., 2013), GST (Rasoulnia et al., 2011), class III peroxidase, catalase and lipoxygenase (Wendelboe-Nelson and Morris, 2012) between differentially drought- and salt-tolerant barleys; in MnSOD, MDH and aconitase between salt-treated wheat and wheat $\times$ Lophopyrum elongatum amphiploid (Jacoby et al., 2013), and a downregulation of $\mathrm{MDH}$ and isocitrate dehydrogenase in coldsensitive spring wheat (Rinalducci et al., 2011a) indicate a crucial role of mitochondrial respiration and ROS metabolism in stress acclimation. Along with these data, a differential abundance in storage proteins such as legumin-like protein between two differentially frost-tolerant winter wheats was found by Vítámvás et al. (2012) indicating a higher demand on energy ensured by storage compound degradation in the less-tolerant genotype. Moreover, tolerant genotypes can also afford to accumulate higher amounts of stress-protective proteins such as PR proteins (Witzel et al., 2014) and ABA-responsive proteins (Alvarez et al., 2014). A significant correlation between wheat WCS120 and barley DHN5 dehydrin relative accumulation and acquired frost tolerance (FT) determined as lethal temperature for $50 \%$ of the sample (LT50) was reported for winter genotypes grown under both cold and moderate cold temperatures (Vítámvás et al., 2007, 2010; Kosová et al., 2008c, 2013c). WCS120 and DHN5 can be thus considered promising FT markers.

Stress-tolerant and stress-sensitive genotypes or related plant species also reveal significant differences in proteins involved in regulation of cell cycle and plant development. Factor eIF5A2 does not only regulate translation inititation, but it is also known to participate in the regulation of cell cycle switch between cell proliferation and death (Thompson et al., 2004). Under salinity, a decreased abundance of eIF5A2 with respect to control was found in both salt-sensitive common wheat and salt-tolerant T. aestivum $\times$ Thinopyrum ponticum hybrid (Wang et al., 2008); however, a decrease in T. aestivum $\times$ Th. ponticum was much lower than in T. aestivum indicating a higher cell proliferation rate in the salttolerant hybrid. A differential abundance in lectin VER2 between cold-treated spring and winter wheat cultivars corresponds to a differential developmental response with a winter wheat revealing a developmental arrest while a spring wheat revealing a progression to reproductive phase as indicated at proteome and phytohormone levels (Kosová et al., 2012, 2013b).

\section{CONCLUSIONS AND FUTURE PERSPECTIVES}

Both abiotic and biotic stress factors induce an active plant stress response including a profound reorganization of plant proteome. Comparative proteomic studies are usually carried out on a limited range of plant material due to their expensiveness and much of sophisticated work. However, they can significantly contribute to identification of novel proteins revealing a differential response in abundance or PTMs between differentially-tolerant genotypes and representing potential protein markers of stress tolerance. The potential markers should be tested on a broad range of genotypes using simple protein quantification methods as ELISA or immunoblots which can be utilized by breeders. As an example, proteomic studies on cold-treated winter wheats resulting in an identification and testing of dehydrin proteins as FT markers can be given (Vítámvás et al., 2007, 2010). Recent publication of draft barley (The International Barley Genome Sequencing Consortium, 2012) and wheat (The International Wheat Genome Sequencing Consortium, 2014) genome sequences will significantly contribute to protein identification, sequentional characterization and preparation of specific antibodies which will stimulate further research and applications in breeding for an improved stress tolerance.

\section{AUTHOR CONTRIBUTIONS}

Klára Kosová has outlined the idea and prepared the text. Pavel Vítámvás and Ilja T. Prášil contributed to preparation, drafting, critical reading, and publication of the manuscript.

\section{ACKNOWLEDGMENTS}

The work was supported by projects from Ministry of Education, Youth and Sports of the Czech Republic (MEYS CZ) LD14064 and LD14087 as a part of of COST actions FA1204 and FA1208, 
respectively. The work was also supported by an institutional project MZE RO0414 from Ministry of Agriculture of the Czech Republic (MZe CZ).

\section{REFERENCES}

Alvarez, S., Choudhury, S. R., and Pandey, S. (2014). Comparative quantitative proteomics analysis of the ABA response of roots of drought-sensitive and drought-tolerant wheat varieties identifies proteomic signatures of drought adaptability. J. Proteome Res. 13, 1688-1701. doi: 10.1021/ pr401165b

Ashoub, A., Beckhaus, T., Berberich, T., Karas, M., and Brüggemann, W. (2013). Comparative analysis of barley leaf proteome as affected by drought stress. Planta 237, 771-781. doi: 10.1007/s00425-012-1798-4

Bahrman, N., Le Gouis, J., Negroni, L., Amilhat, L., Leroy, P., Lainé, A. L., et al. (2004). Differential protein expression assessed by two-dimensional gel electrophoresis for two wheat varieties grown at four nitrogen levels. Proteomics 4, 709-719. doi: 10.1002/pmic.200300571

Brini, F., Hanin, M., Lumbreras, V., Irar, S., Pagès, M., and Masmoudi, K. (2007). Functional characterization of DHN-5, a dehydrin showing a differential phosphorylation pattern in two Tunisian durum wheat (Triticum durum Desf.) varieties with marked differences in salt and drought tolerance. Plant Sci. 172, 20-28. doi: 10.1016/j.plantsci.2006.07.011

Budak, H., Akpinar, B. A., Unver, T., and Turktas, M. (2013). Proteome changes in wild and modern wheat leaves upon drought stress by two-dimensional electrophoresis and nanoLC-ESI-MS/MS. Plant Mol. Biol. 83, 89-103. doi: 10.1007/s11103-013-0024-5

Caruso, G., Cavaliere, C., Foglia, P., Gubbiotti, R., Samperi, R., and Laganà, A. (2009). Analysis of drought responsive proteins in wheat (Triticum durum) by 2D-PAGE and MALDI-TOF mass spectrometry. Plant Sci. 177, 570-576. doi: 10.1016/j.plantsci.2009.08.007

Caruso, G., Cavaliere, C., Guarino, C., Gubbiotti, R., Foglia, P., and Laganà, A. (2008). Identification of changes in Triticum durum L. leaf proteome in response to salt stress by two-dimensional electrophoresis and MALDI-TOF mass spectrometry. Anal. Bioanal. Chem. 391, 381-390. doi: 10.1007/s00216008-2008-x

Cattivelli, L., Rizza, F., Badeck, F. W., Mazzucotelli, E., Mastrangelo, A. M., Francia, E., et al. (2008). Drought tolerance improvement in crop plants: An integrated view from breeding to genomics. Field Crops Res. 105, 1-14. doi: 10.1016/j.fcr.2007.07.004

Crosatti, C., Soncini, C., Stanca, A. M., and Cattivelli, L. (1995). The accumualtion of a cold-regulated chloroplastic protein is light dependent. Planta 195 $458-463$.

Edreva, A. (2005). Pathogenesis-related proteins: research progress in the last 15 years. Gen. Appl. Plant Physiol. 31, 105-124.

Eggert, K., and Pawelzik, E. (2011). Proteome analysis of Fusarium head blight in grains of naked barley (Hordeum vulgare subsp. nudum). Proteomics 11, 972-985. doi: 10.1002/pmic.201000322

Eggert, K., Zörb, C., Mühling, K. H., and Pawelzik, E. (2011). Proteome analysis of Fusarium infection in emmer grains (Triticum dicoccum). Plant Pathol. 60, 918-928. doi: 10.1111/j.1365-3059.2011.02442.x

Fatehi, F., Hosseinzadeh, A., Alizadeh, H., Brimavandi, T., and Struik, P. C. (2012). The proteome response of salt-resistant and salt-sensitive barley genotypes to long-term salinity stress. Mol. Biol. Rep. 39, 6387-6397. doi: 10.1007/s11033012-1460-z

Fercha, A., Capriotti, A. L., Caruso, G., Cavaliere, C., Gherroucha, H., Samperi, R., et al. (2013). Gel-free proteomics reveal potential biomarkers of priminginduced salt tolerance in durum wheat. J. Proteomics 91, 486-499. doi: 10.1016/j.jprot.2013.08.010

Fercha, A., Capriotti, A. L., Caruso, G., Cavaliere, C., Samperi, R. Stampachiacchiere, S., et al. (2014). Comparative analysis of metabolic proteome variation in ascorbate-primed and unprimed wheat seeds during germination under salt stress. J. Proteomics 108, 238-257. doi: 10.1016/j.jprot.2014.04.040

Ford, K. L., Cassin, A., and Bacic, A. (2011). Quantitative proteomic analysis of wheat cultivars with differing drought stress tolerance. Front. Plant. Sci. 2:44. doi: 10.3389/fpls.2011.00044

Ghabooli, M., Khatabi, B., Ahmadi, F. S., Sepehri, M., Mizraei, M., Amirkhani, A., et al. (2013). Proteomics study reveals the molecular mechanisms underlying water stress tolerance induced by Piriformospora indica in barley. J. Proteomics 94, 289-301. doi: 10.1016/j.jprot.2013.09.017

Gharechahi, J., Alizadeh, H., Reza Naghavi, M., and Sharifi, G. (2014). A proteomic analysis to identify cold acclimation associated proteins in wild wheat (Triticum urartu L.). Mol. Biol. Rep. 41, 3897-3905. doi: 10.1007/s11033-0143257-8

Gonzalez-Fernandez, R., and Jorrin-Novo, J. V. (2012). Contribution of proteomics to the study of plant pathogenic fungi. J. Proteome Res. 11, 3-16. doi: $10.1021 / \mathrm{pr} 200873 \mathrm{p}$

Hajheidari, M., Eivazi, A., Buchanan, B. B., Wong, J. H., Majidi, I., and Salekdeh, G. H. (2007). Proteomics uncovers a role for redox in drought tolerance in wheat. J. Proteome Res. 6, 1451-1460. doi: 10.1021/pr060570j

Hlaváèková, I., Vítámvás, P., Šantrůček, J., Kosová, K., Zelenková, S., Prášil, I. T. et al. (2013). Proteins involved in distinct phases of cold hardening process in frost resistant winter barley (Hordeum vulgare L.) cv. Luxor. Int. J. Mol. Sci. 44, 8000-8024. doi: 10.3390/ijms14048000

Hossain, Z., Nouri, M. Z., and Komatsu, S. (2012). Plant cell organelle proteomics in response to abiotic stress. J. Proteome Res. 11, 37-48. doi: 10.1021/ pr200863r

Jacoby, R. P., Millar, A. H., and Taylor, N. L. (2010). Wheat mitochondrial proteomes provide new links between antioxidant defense and plant salinity tolerance. J. Proteome Res. 9, 6595-6604. doi: 10.1021/pr1007834

Jacoby, R. P., Millar, A. H., and Taylor, N. L. (2013). Investigating the role of respiration in plant salinity tolerance by analyzing mitochondrial proteomes from wheat and a salinity-tolerant amphiploid (Wheat $\times$ Lophopyrum elongatum). J. Proteome Res. 12, 4807-4829. doi: 10.1021/pr400504a

Jorrin-Novo, J. V., Maldonado, A. M., Echavarría-Zomeno, S., Valledor, L., Castillejo, M. A., Curto, M., et al. (2009). Plant proteomics update (20072008): second-generation proteomic techniques, an appropriate experimental design, and data analysis to fulfill MIAPE standards, increase plant proteome coverage and expand biological knowledge. J. Proteomics 72, 285-314. doi: 10.1016/j.jprot.2009.01.026

Kamal, A. H. M., Cho, K., Kim, D. E., Uozumi, N., Chung, K. Y., Lee, S. Y., et al. (2012). Changes in physiology and protein abundance in saltstressed wheat chloroplasts. Mol. Biol. Rep. 39, 9059-9074. doi: 10.1007/s11033012-1777-7

Kang, G., Li, G., Xu, W., Peng, X., Han, Q., Zhu, Y., et al. (2012). Proteomics reveals the effects of salicylic acid on growth and tolerance to subsequent drought stress in wheat. J. Proteome Res. 11, 6066-6079. doi: 10.1021/pr300728y

Kosová, K., Chrpová, J., and Šíp, V. (2008b). Recent advances in breeding of cereals for resistance to barley yellow Dwarf virus - a review. Czech J. Genet. Plant Breed. $44,1-10$.

Kosová, K., Chrpová, J., and Šíp, V. (2009). Cereal resistance to Fusarium head blight and possibilities of its improvement through breeding. Czech J. Genet. Plant Breed. 45, 87-105.

Kosová, K., Holková, L., Prášil, I. T., Prášilová, P., Bradáčová, M., Vítámvás, P., et al. (2008c). Expression of dehydrin 5 during the development of frost tolerance in barley (Hordeum vulgare). J. Plant Physiol. 165, 1142-1151. doi: 10.1016/j.jplph.2007.10.009

Kosová, K., Prášil, I. T., and Vítámvás, P. (2008a). The relationship between vernalization- and photoperiodically-regulated genes and the development of frost tolerance in wheat and barley. Biol. Plant. 52, 601-615. doi: 10.1007/s10535-008-0120-6

Kosová, K., Prášil, I. T., and Vítámvás, P. (2013a). Protein contribution to plant salinity response and tolerance acquisition. Int. J. Mol. Sci. 14, 6757-6789. doi: 10.3390/ijms14046757

Kosová, K., Prášil, I. T., Vítámvás, P., Dobrev, P., Motyka, V., Floková, K., et al. (2012). Complex phytohormone responses during the cold acclimation of two wheat cultivars differing in cold tolerance, winter Samanta and spring Sandra. J. Plant Physiol. 169, 567-576. doi: 10.1016/j.jplph.2011.12.013

Kosová, K., Vítámvás, P., Planchon, S., Renaut, J., Vanková, R., and Prášil, I. T. (2013b). Proteome analysis of cold response in spring and winter wheat (Triticum aestivum) crowns reveals similarities in stress adaptation and differences in regulatory processes between the growth habits. J. Proteome Res. 12, 4830-4845. doi: 10.1021/pr400600g

Kosová, K., Vítámvás, P., Prášil, I. T., and Renaut, J. (2011). Plant proteome changes under abiotic stress-contribution of proteomics studies to understanding plant stress response. J. Proteomics 74, 1301-1322. doi: 10.1016/j.jprot.2011.02.006 
Kosová, K., Vítámvás, P., Prášilová, P., and Prášil, I. T. (2013c). Accumulation of WCS120 and DHN5 proteins in differently frost-tolerant wheat and barley cultivars grown under a broad temperature scale. Biol. Plant. 57, 105-112. doi: 10.1007/s10535-012-0237-5

Labhilili, M., Joudrier, P., and Gautier, M. F. (1995). Characterization of cDNAs encoding Triticum durum dehydrins and their expression patterns in cultivars that differ in drought tolerance. Plant Sci. 112, 219-230. doi: 10.1016/01689452(95)04267-9

Larcher, W. (2003). Physiological Plant Ecology, 4th Edn. Berlin; Heidelberg; Springer Verlag.

Levitt, J. (1980). Responses of Plants to Environmental Stress. Chilling, Freezing and High Temperature Stresses, 2nd Edn. New York, NY: Academic Press.

Li, G., Peng, X., Xuan, H., Wei, L., Yang, Y., Guo, T., et al. (2013). Proteomic analysis of leaves and roots of common wheat (Triticum aestivum $\mathrm{L}$.) under copper-stress conditions. J. Proteome Res. 12, 4846-4861. doi: 10.1021/pr4008283

Li, X., Cai, J., Liu, F., Dai, T., Cao, W., and Jiang, D. (2014). Physiological, proteomic and transcriptional responses of wheat to combination of drought or waterlogging with late spring low temperature. Funct. Plant Biol. 41, 690-703. doi: 10.1071/FP13306

Majoul, T., Bancel, E., Triboi, E., Ben Hamida, J., and Branlard, G. (2004). Proteomic analysis of the effect of heat stress on hexaploid wheat grain: Characterization of heat-responsive proteins from non-prolamins fraction. Proteomics 4, 505-513. doi: 10.1002/pmic.200300570

Mittler, R. (2006). Abiotic stress, the field environment and stress combination. Trends Plant Sci. 11, 15-19. doi: 10.1016/j.tplants.2005.11.002

Mori, S., and Nishizawa, N. (1987). Methionine as a dominant precursor of phytosiderophores in Graminaceae plants. Plant Cell Physiol. 28, 1081-1092.

Munns, R. (2005). Genes and salt tolerance: bringing them together. New Phytol. 167, 645-663. doi: 10.1111/j.1469-8137.2005.01487.x

Patterson, J., Ford, K., Cassin, A., Natera, S., and Bacic, A. (2007). Increased abundance of proteins involved in phytosiderophore production in boron-tolerant barley. Plant Physiol. 144, 1612-1631. doi: 10.1104/pp.107.096388

Peng, Z., Wang, M., Li, F., Lv, H., Li, C., and Xia, G. (2009). A proteomic study of the response to salinity and drought stress in an introgression strain of bread wheat. Mol. Cell. Proteomics 8, 2676-2686. doi: 10.1074/mcp.M900052MCP200

Rampitsch, C., Bykova, N. V., McCallum, B., Beimcik, E., and Ens, W. (2006). Analysis of the wheat and Puccinia triticina (leaf rust) proteomes during a susceptible host-pathogen interaction. Proteomics 6, 1897-1907. doi: 10.1002/pmic.200500351

Rasoulnia, A., Bihamta, M. R., Peyghambari, S. A., Alizadeh, H., and Rahnama, A. (2011). Proteomic response of barley leaves to salinity. Mol. Biol. Rep. 38 , 5055-5063. doi: 10.1007/s11033-010-0651-8

Rinalducci, S., Egidi, K. G., Mahfoozi, S., Godehkahriz, S. J., and Zolla, L. (2011b). The influence of temperature on plant development in a vernalization-requiring winter wheat: A 2-DE based proteomic investigation. J. Proteomics 74, 643-659. doi: 10.1016/j.jprot.2011.02.005

Rinalducci, S., Egidi, M. G., Karimzadeh, G., Jazii, F., and Zolla, L. (2011a). Proteomic analysis of a spring wheat cultivar in response to prolonged cold stress. Electrophoresis 32, 1807-1818. doi: 10.1002/elps.201000663

Rollins, J. A., Habte, E., Templer, S. E., Colby, T., Schmidt, J., and von Korff, M. (2013). Leaf proteome alterations in the context of physiological and morphological responses to drought and heat stress in barley (Hordeum vulgare L.). J. Exp. Bot. 64, 3201-3212. doi: 10.1093/jxb/ert158

Sarhadi, E., Mahfoozi, S., Hosseini, S. A., and Salekdeh, G. H. (2010). Cold accliamtion proteome analysis reveals close link between upregulation of lowtemperature associated proteins and vernalization fulfillment. J. Proteome Res. 9, 5658-5667. doi: 10.1021/pr100475r

Sergeant, K., and Renaut, J. (2010). Plant biotic stress and proteomics. Curr. Proteomics 7, 275-297. doi: 10.2174/157016410793611765

Skylas, D. J., Cordwell, S. J., Hains, P. G., Larsen, M. R., Basseal, D. J., Walsch, B. J., et al. (2002). Heat shock of wheat during grain filling: Proteins associated with heat tolerance. J. Cereal Sci. 35, 175-188. doi: 10.1006/jcrs.2001.0410

Sugimoto, M., and Takeda, K. (2009). Proteomic analysis of specific proteins in the root of salt-tolerant barley. Biosci. Biotechnol. Biochem. 73, 2762-2765. doi: 10.1271/bbb. 90456

The International Barley Genome Sequencing Consortium. (2012). A physical, genetic and functional sequence assembly of the barley genome. Nature 491, 711-717. doi: 10.1038/nature11543
The International Wheat Genome Sequencing Consortium. (2014). Slicing the wheat genome. Science 345, 285-287. doi: 10.1126/science

Thomashow, M. F. (1999). Plant cold acclimation. Freezing tolerance genes and regulatory mechanisms. Annu. Rev. Plant Physiol. Plant Mol. Biol. 50, 571-599. doi: 10.1146/annurev.arplant.50.1.571

Thompson, J. E., Hopkins, M. E., Taylor, C., and Wang, T. W. (2004). Regulation of senescence by eukaryotic translation initiation factor 5A: implications for plant growth and development. Trends Plant Sci. 9, 174-179. doi: 10.1016/j.tplants.2004.02.008

Vágújfalvi, A., Crosatti, C., Galiba, G., Dubcovsky, J., and Cattivelli, L. (2000). Two loci of chromosome $5 \mathrm{~A}$ regulate the differential cold-dependent expression of the cor-14b gene in frost-tolerant and frost-sensitive genotypes. Mol. Gen. Genet. 263, 194-200. doi: 10.1007/s004380051160

Vágújfalvi, A., Galiba, G., Cattivelli, L., and Dubcovsky, J. (2003). The coldregulated transcriptional activator $\mathrm{Cbf3}$ is linked to the frost-tolerance locus Fr-A2 on wheat chromosome 5A. Mol. Gen. Genomics 269, 60-67. doi: 10.1007/s00438-003-0806-6

Vítámvás, P., Kosová, K., Prášilová, P., and Prášil, I. T. (2010). Accumulation of WCS120 protein in wheat cultivars grown at $9^{\circ} \mathrm{C}$ or $17^{\circ} \mathrm{C}$ in relation to their winter survival. Plant Breed. 129, 611-616. doi: 10.1111/j.14390523.2010.01783.x

Vítámvás, P., Prášil, I. T., Kosová, K., Planchon, S., and Renaut, J. (2012). Analysis of proteome and frost tolerance in chromosome $5 \mathrm{~A}$ and $5 \mathrm{~B}$ reciprocal substitution lines between two winter wheats during long-term cold acclimation. Proteomics 12, 68-85. doi: 10.1002/pmic.201000779

Vítámvás, P., Saalbach, G., Prášil, I. T., Čapková, V., Opatrná, J., and Jahoor, A. (2007). WCS120 protein family and proteins soluble upon boiling in cold-acclimated winter wheat. J. Plant Physiol. 164, 1197-1207. doi: 10.1016/j.jplph.2006.06.011

Wang, M. C., Peng, Z. Y., Li, C. L., Li, F., Liu, C., and Xia, G. M. (2008). Proteomic analysis on a high salt tolerance introgression strain of Triticum aestivum/Thinopyrum ponticum. Proteomics 8, 1470-1489. doi: 10.1002/pmic. 200700569

Wendelboe-Nelson, C., and Morris, P. C. (2012). Proteins linked to drought tolerance revealed by DIGE analysis of drought resistant and susceptible barley varieties. Proteomics 12, 3374-3385. doi: 10.1002/pmic.201200154

Witzel, K., Matros, A., Strickert, M., Kaspar, S., Peukert, M., Mühling, K. H., et al. (2014). Salinity stress in roots of contrasting barley genotypes reveals time-distinct and genotype-specific patterns for defined proteins. Mol. Plant 7, 336-355. doi: 10.1093/mp/sst063

Witzel, K., Weidner, A., Surabhi, G. K., Börner, A., and Mock, H. P. (2009). Salt stress-induced alterations in the root proteome of barley genotypes with contrasting response towards salinity. J. Exp. Bot. 60, 3545-3557. doi: 10.1093/jxb/erp198

Xu, J., Li, Y., Sun, J., Du, L., Zhang, Y., Yu, Q., et al. (2013). Comparative physiological and proteomic response to abrupt low temperature stress in two winter wheat cultivars differing in low temperature tolerance. Plant Biol. 15, 292-303. doi: 10.1111/j.1438-8677.2012.00639.x

Yang, F., Jensen, J. D., Spliid, N. H., Svensson, B., Jacobsen, S., Jørgensen, L. N., et al. (2010b). Investigation of the effect of nitrogen on severity of Fusarium Head Blight in barley. J. Proteomics 73, 743-752. doi: 10.1016/j. jprot.2009

Yang, F., Jensen, J. D., Svensson, B., Jørgensen, H. J. L., Collinge, D. B., and Finnie, C. (2010a). Analysis of early events in the interaction between Fusarium graminearum and the susceptible barley (Hordeum vulgare) cultivar Scarlett. Proteomics 10, 3748-3755. doi: 10.1002/pmic.201000243

Yang, F., Melo-Braga, M. N., Larsen, M. R., Jørgensen, H. J. L., and Palmisano, G. (2013). Battle through signaling between wheat and the fungal pathogen Septoria tritici revealed by proteomics and phosphoproteomics. Mol. Cell. Proteomics 12, 2497-2508. doi: 10.1074/mcp.M113.027532

Ye, J., Wang, S., Zhang, F., Xie, D., and Yao, Y. (2013). Proteomic analysis of leaves of different wheat genotypes subjected to PEG6000 stress and rewatering. Plant OMICS J. 6, 286-294

Yong, W. D., Xu, Y. Y., Xu, W. Z., Wang, X., Li, N., Wu, J. S., et al. (2003). Vernalization-induced flowering in wheat is mediated by a lectin-like gene VER2. Planta 217, 261-270. doi: 10.1007/s00425-003-0994-7

Zhang, H., Han, B., Wang, T., Chen, S., Li, H., Zhang, Y., et al. (2012). Mechanisms of plant stress response: Insights from proteomics. J. Proteome Res. 11, 49-67. doi: $10.1021 / p r 200861 w$ 
Zhang, M., Lv, D., Ge, P., Bian, Y., Chen, G., Zhu, G., et al. (2014). Phosphoproteome analysis reveals new drought response and defense mechanisms of seedling leaves in bread wheat (Triticum aestivum L.). J. Proteomics 109, 290-308. doi: 10.1016/j.jprot.2014.07.010

Conflict of Interest Statement: The authors declare that the research was conducted in the absence of any commercial or financial relationships that could be construed as a potential conflict of interest.

Received: 15 October 2014; accepted: 26 November 2014; published online: 11 December 2014.
Citation: Kosová K, Vitámvás P and Prášil IT (2014) Proteomics of stress responses in wheat and barley-search for potential protein markers of stress tolerance. Front. Plant Sci. 5:711. doi: 10.3389/fpls.2014.00711

This article was submitted to Plant Proteomics, a section of the journal Frontiers in Plant Science.

Copyright (C) 2014 Kosová, Vítámvás and Prášil. This is an open-access article distributed under the terms of the Creative Commons Attribution License (CC BY). The use, distribution or reproduction in other forums is permitted, provided the original author(s) or licensor are credited and that the original publication in this journal is cited, in accordance with accepted academic practice. No use, distribution or reproduction is permitted which does not comply with these terms. 